\title{
Metastatic Signet-Ring Cell Gastric Carcinoma Masquerading as Breast Primary
}

\author{
Dinesh Chandra Doval ${ }^{a}$ Shripad Brahmanand Pande \\ Jai Bhagwan Sharma ${ }^{a}$ Sameer Khatri ${ }^{a}$ \\ Arvind Kumar Khurana ${ }^{\mathrm{b}} \quad$ Ashok Kumar Vaid $^{\mathrm{a}}$ \\ Arvind Kumar Chaturvedic Digant Hazarika ${ }^{d}$
}

Departments of a Medical Oncology, ${ }^{b}$ Internal Medicine, Gastroenterology Subspecialty, 'Radiodiagnosis and ${ }^{\mathrm{d} P a t h o l o g y, ~ R a j i v ~ G a n d h i ~ C a n c e r ~ I n s t i t u t e ~ a n d ~}$ Research Center, New Delhi, India

\section{Key Words}

Breast metastases $\cdot$ Krukenberg $\cdot$ Signet-ring cell carcinoma $\cdot$ Gastric carcinoma

\begin{abstract}
Metastasis to the breast from an extra-mammary primary is a rare phenomenon; metastasis from gastric carcinoma to the breast is extremely so. We report a case who initially presented as mucin-secreting and signet-ring cell tumor of the ovary, and after an interval of 8 months with breast and chest wall metastatic nodules. The covert gastric primary eluded the oncologists at both presentations.
\end{abstract}

\section{Introduction}

Extra-mammary tumors that most frequently metastasize to the breast are melanomas and lymphomas [1]. Signet-ring cell carcinoma metastatic to the breast is a rare occurrence; signet-ring cell carcinoma of gastrointestinal origin, especially the stomach, metastasizing to the breast has occasionally been reported $[2,3]$.

\section{Case Report}

A 34-year-old lady with no significant comorbidities had been investigated elsewhere (i.e. at another center) for complaints of low backache and abdominal distension in June 2004. Bilateral adnexal masses had been seen on abdominal ultrasound. CA 125 was $109.67 \mathrm{U} / \mathrm{ml}$. She had undergone total abdominal hysterectomy with bilateral salpingo-oophorectomy in the last week of June 2004 at a Medical College 
Hospital in the sub-Himalayan region. Histopathological examination had suggested mucin-secreting and signet-ring cell adenocarcinoma of the ovaries. After a futile search for the identification of gastrointestinal primary, especially of gastric origin (with endoscopy), a diagnosis of Krukenberg tumor of bilateral ovaries of 'probable' gastrointestinal origin was made. Postoperatively she had developed abdominal distension and ascites and had consequently been given chemotherapy based on oxaliplatin and capcitabine at the same center for 6 cycles from September 2004.

After an interval of about 8 months of initial diagnosis, she was referred to us. She complained of bilateral breast lumps, nodules in the skin overlying the breasts, gradually increasing breathlessness and distension of abdomen of two months' duration. On examination, she was found to have enlarged and hard right supraclavicular lymph node, right axillary lymph nodes, and induration of both breasts and nodules in the skin overlying the breasts (ig. 1). In addition, she had bilateral pleural effusions and ascites.

With a provisional working impression of breast cancer, we probed her further. Hemogram was normal, biochemistry revealed hypoproteinemia and hypoalbuminemia. CA 125 was $64 \mathrm{U} / \mathrm{ml}$ and CA 15-3 was $18.4 \mathrm{U} / \mathrm{ml}$ (normal $<35 \mathrm{U} / \mathrm{ml}$ ). Chest X-ray showed obliteration of both cardiophrenic angles. Haziness was noted in both the right and left mid and lower zones. Diffuse increased density and skin thickening without an obvious mass lesion was documented on mammography. Computed tomography $(\mathrm{CT})$ of the abdomen showed gastric wall thickening with infiltration of perigastric fat (fig. 2), ascites, and lytic lesions in multiple vertebrae, the pelvic bones and the right femur. Bone scan confirmed metastases in $\mathrm{D}_{11}$ through $\mathrm{L}_{2}$ vertebrae and the neck of the right femur.

A Tru-cut biopsy from the left breast lesion showed malignant cells in small clusters with signet ring appearance. Immunostaining was positive for cytokeratin, epithelial membrane antigen, and was negative for gross cystic fluid protein (GCDFP-15), ER, PR, Her-2/neu (ERBB2) and CA 125. This immunohistochemistry array supported the diagnosis of signet-ring cell carcinoma in the breast of metastatic nature rather than of primary kind. Pleural fluid cytology was negative for malignant cells.

Directed by her symptoms referable to the alimentary tract and by the CT scan discovery of stomach wall thickening, we performed esophagogastroduodenoscopy (EGD); nodular, ulcerated and friable mucosa of the cardia and the proximal body (fig. 3 ) of the stomach was observed. Biopsy reported as signet-ring cell carcinoma with a glandular arrangement (ig. $)$. A final diagnosis of cancer of the stomach metastatic to the breasts, lung, bone, supraclavicular and axillary lymph nodes and skin (as subcutaneous nodules) was entertained.

After thorough discussion with the family, she was started on docetaxel and cisplatin-based palliative chemotherapy, of which two cycles were administered. In the aftermath of the 2 nd cycle of chemotherapy she required supportive care. In view of the progressive nature of the disease, she was discharged on request with advice to continue supportive treatment at a Hospice.

\section{Discussion}

An extra-mammary neoplasm metastasizing to the breast is a rare occurrence. The incidence of this phenomenon ranges from 1.7 to $6.6 \%$ in autopsy series, from 1.2 to $2 \%$ in clinical reports and to $2.7 \%$ in cytological series $[3,4]$. Tumors that metastasize to the breast most frequently are melanomas and lymphomas, followed by cancers of the lung, ovary, kidney and stomach [5].

Primary signet-ring cell carcinoma of the breast occurs rarely, as described by Merino and Livolsi [6] and Harris et al. [7]. It has to be distinguished from metastatic signet-ring cell carcinoma of extra-mammary origin. It is this distinction that is critical for the appropriate management. Immunohistochemistry usually helps discriminate primary from metastatic signet-ring cell carcinoma, as primary breast signet-ring cell carcinoma most often is immunoreactive to GCDFP-15, and is estrogen receptor and progesterone receptor positive [8].

Signet-ring cell carcinoma metastatic to the breast is a rare occurrence; only a few cases have been reported $[2,3,8-10]$. The first case of metastasis to the breast from gastric 
signet-ring cell carcinoma was described by Dawson in 1936 [9], whereas a case with metastatic localization of signet-ring cell gastric carcinoma to the breast and ovaries (the breast-ovary-gastric carcinoma association) was reported by Di Cosimo et al. in 2003 [10].

Signet-ring cell carcinoma of the stomach is characterized by younger age at presentation and female preponderance [11] and by fulminant and rapidly progressive course exemplified by our case. The growth of gastric cancer may be influenced by the natural biological and hormonal milieu [12]. We emphasize that in signet-ring cell histology of the breast, discrimination of a primary breast neoplasm from a nonmammary neoplasm metastasizing to the breast is critical for optimal management. In our case, the diagnosis of secondary breast involvement was supported by not just the immunohistochemical display. It was also supported by the CT and endoscopic discovery of the stomach primary. Biopsy from the stomach clenched the diagnosis of gastric signet-ring cell carcinoma.

The case under discussion was cryptic to the previous oncologist, who treated the Krukenberg tumor, ascribing it to a gastrointestinal origin, but his attempt at finding the gastrointestinal primary was futile. To the authors, who treated the patient subsequently, the case presented a challenge until the substantiation of the metastatic (rather than primary) nature of the breast lesions came in from the immunohistochemistry of the breast biopsy and the documentation of gastric primary on EGD and gastric biopsy.

\section{Conclusion}

It is a well-known fact that signet-ring cell carcinoma of the stomach frequently presents as Krukenberg tumor. We present a case of gastric carcinoma that masqueraded initially as ovarian tumor and then, at recurrence, as primary neoplasm of the breast. Until its eventual uncovering, the covert gastric primary eluded the physicians on both occasions.

Fig. 1. Photograph showing bilateral breast lesions and skin nodules.

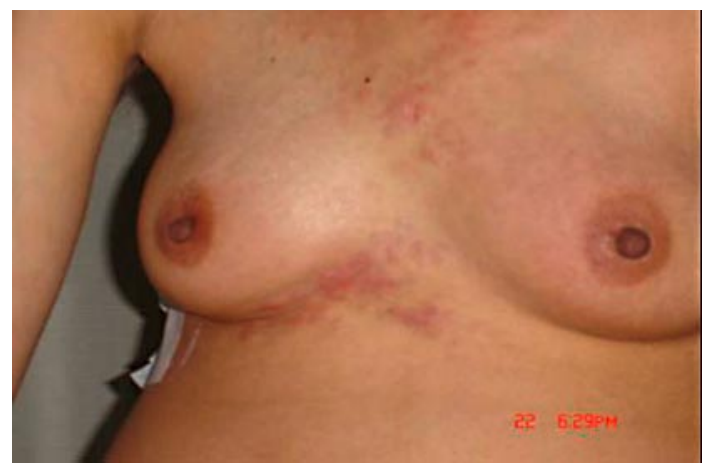


Fig. 2. CT scan of the abdomen showing gastric wall thickening with infiltration of perigastric fat.

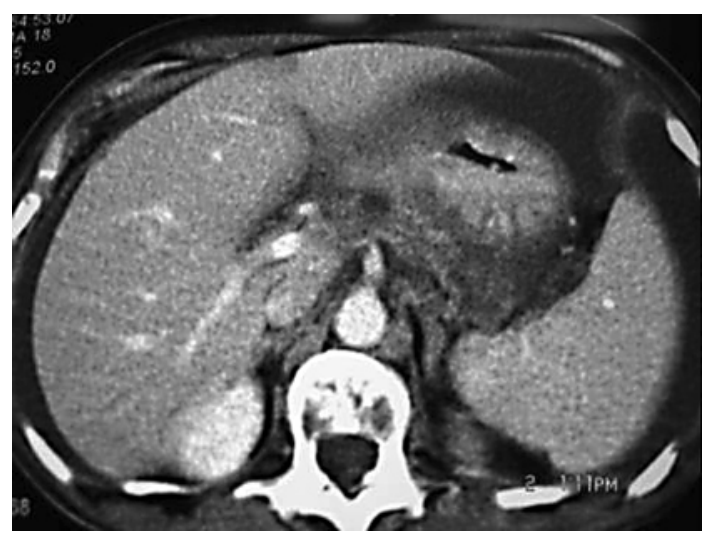

Fig. 3. Upper gastrointestinal endoscopy (EGD) showing thickened friable mucosa of the body of the stomach.

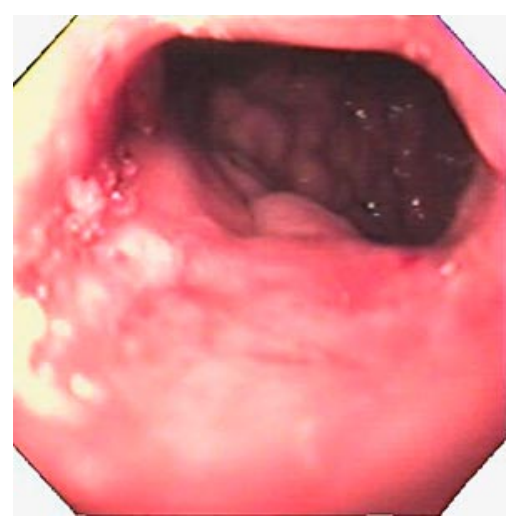

Fig. 4. Histology of breast lesion showing tumor cells with signet-ring configuration due to mucin secretion in the cytoplasm pushing the nucleus to the periphery, close to the cell membrane.

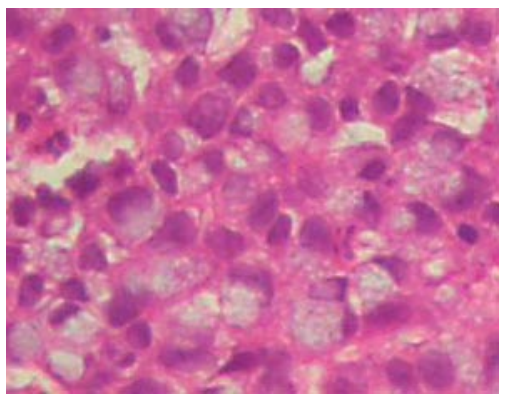




\section{References}

1 Paulus DD, Libshitz HI: Metastasis to the breast. Radiol Clin North Am 1982;20:561-568.

2 Kwak JY, Kim EK, Oh KK: Radiologic findings of metastatic signet ring cell carcinoma to the breast from stomach. Yonsei Med J 2000;41:669-672.

-3 Cavazzini G, Colpani F, Cantore M, et al: Breast metastasis from gastric signet ring cell carcinoma, mimicking inflammatory carcinoma. A case report. Tumori 1993;79:450-453.

4 Alva S, Shetty-Alva N: An update of tumor metastasis to the breast data. Arch Surg 1999;134:450.

-5 Shukla R, Pooja B, Radhika S, Nijhawan R, Rajwanshi A: Fine-needle aspiration cytology of extramammary neoplasms metastatic to the breast. Diagn Cytopathol 2005;32:193-197.

6 Merino MJ, Livolsi VA: Signet ring carcinoma of the female breast: a clinicopathologic analysis of 24 cases. Cancer 1981;48:1830-1837.

7 Harris M, Wells S, Vasudev KS: Primary signet ring cell carcinoma of the breast. Histopathology 1978;2:171-176.

8 Qureshi SS, Shrikhande SV, Tanuja S, Shukla PJ: Breast metastases of gastric signet ring cell carcinoma. A differential diagnosis with primary breast signet ring cell carcinoma. J Postgrad Med 2005;51:125-127.

-9 Dawson EK: Metastatic tumour of breast, with report of a case. J Pathol Bacteriol 1936;43:53-60.

10 Di Cosimo S, Ferretti G, Fazio N, et al: Breast and ovarian metastatic localization of signet ring cell gastric carcinoma. Ann Oncol 2003;14:803-804.

11 Antonioli DA, Goldman H: Changes in the location and type of gastric adenocarcinoma. Cancer 1982;50:775-781.

12 Maeta M, Yamashiro H, Oka A, et al: Gastric cancer in the young, with special reference to 14 pregnancy associated cases: analysis based on 2,325 consecutive cases of gastric cancer. J Surg Oncol 1995;58:191-195. 